\title{
Correspondence
}

Epidemiol. Infect. (2012).

doi:10.1017/S0950268812002051

First published online 25 September 2012

\section{Microbiological and clinical characteristics of bacteraemia caused by the hypermucoviscosity phenotype of Klebsiella pneumoniae in Korea}

To the Editor

We read with great interest the recently published article by Jung et al. [1]. The authors identified one ST380 serotype K2 blood isolate with liver abscess as the source of bacteraemia. We have recently reported three fatal bacteraemia episodes due to this clone in France [2]. However, these episodes were of unknown origin or related to pneumonia. To highlight the medical interest of data provided by Jung et al., we report the diagnosis in France of a liver abscess due to ST380, as well as one liver abscess case caused by a K2 isolate of ST375. The latter sequence type was also identified by Jung et al., but not from a liver abscess. The patient infected by isolate ST380 was a 68-yearold Tunisian man who had worked in France. He was hospitalized in France in order to explore a heterogeneous hepatic mass that was identified by echography when he visited Tatouine hospital in Tunisia for fever and chills. The hepatic abscess was complicated by a bilio-digestive fistula. The patient recovered following abscess and biliary drainage and amoxicillin + clavulanate antibiotic treatment. The second patient was a French woman with a 1-week history of fever and fatigue. She was a native of France, had no significant medical history and denied alcohol or drug abuse. She had travelled in Vietnam 2 years previously. Clinical examination showed a temperature of $40{ }^{\circ} \mathrm{C}$ and tenderness to percussion over the right upper quadrant of the abdomen. The remainder of the physical examination was unremarkable. Abdominal computed tomography showed a 10-cm diameter liver abscess. The patient recovered after abscess drainage and treatment with cefotaxime. Moreover, as with Jung et al. we detected the $k f u$ gene, previously identified in non-K2 strains causing hepatic and extrahepatic abscesses, in strain ST380 but not in strain ST375 (PCR primers: 5'-AGAACCTTCCTCGCTGAACA-3' and 5'-ATAGTAGGCGAGCACCGAGA-3'). In addition to the already recognized ST23-ST57 K 1 clone, these results underline the emergence of at least two K. pneumoniae clones of capsular serotype K2, ST380 and ST375, which can cause liver abscess, and further emphasize the invasive potential of these two clones. Further studies are required to investigate the virulence traits of $\mathrm{K} 2$ strains.

\section{Declaration of Interest}

None.

\section{References}

1. Jung SW, et al. Microbiological and clinical characteristics of bacteraemia caused by the hypermucoviscosity phenotype of Klebsiella pneumoniae in Korea. Epidemiology and Infection. Published online: 14 May 2012. doi:10.1017/S0950268812000933.

2. Decré $\mathbf{D}$, et al. Emerging severe and fatal infections due to Klebsiella pneumoniae in two university hospitals in France. Journal of Clinical Microbiology 2011; 49: 3012-3014.

S UZANNE B I A LEK - D A VENET $T^{1,2}$, MARIE-HÉLÈNE NICOLAS-CHANOIN E DOMINIQUE DECRÉ ${ }^{3}$, SYLVAIN BRISSE ${ }^{4}$

${ }^{1}$ Hôpital Beaujon, AP-HP, Clichy, France

${ }^{2}$ Inserm CRB3 U773, Paris, France

${ }^{3}$ Hopital Saint Antoine, AP-HP, Paris, France

${ }^{4}$ Institut Pasteur, Paris, France

(Email: mhn.chanoine@bjn.aphp.fr) 